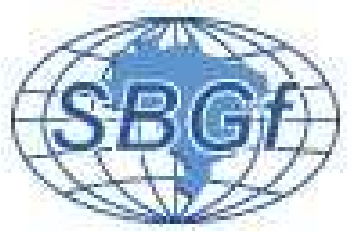

\title{
Processamento, Inversão e Imageamento de Dados Sísmicos Marinhos da Bacia do Jequitinhonha
}

Silva, D. A. B; Leite, L. W. B. Universidade Federal do Pará

Copyright 2012, SBGf - Sociedade Brasileira de Geofísica. Este texto foi preparado para a apresentação no V Simpósio Brasileiro de Geofísica, Salvador, 27 a 29 de novembro de 2012. Seu conteúdo foi revisado pelo Comitê Técnico do V SimBGf, mas não necessariamente representa a opinião da SBGf ou de seus associados. É proibida a reprodução total ou parcial deste material para propósitos comerciais sem prévia autorização da SBGf.

\section{RESUMO}

Este trabalho é voltado ao processamento, inversão e imageamento da linha L2140266 selecionada da bacia sedimentar do Jequitinhonha, Bahia, adquirida pela PETROBRAS, e baseia-se na aplicação das metodologias de empilhamento $\mathrm{NMO} / \mathrm{CRS}$ e na inversão tomográfica da onda NIP, onde a migração do tipo Kirchhoff pósempilhamento, no tempo e na profundidade, é comum a todas as técnicas. Além disso, foi aplicado o empilhamento CRS-parcial para densificação das famílias CMPs. Vários testes foram realizados para otimizar parâmetros, aberturas dos operadores, minimizar a função objetivo e regularizar os parâmetros do modelo, e como resultado foram escolhidos os fluxogramas de processamento que geraram melhor qualidade visual das seções, com os principais resultados comparados no decorrer do trabalho para ambas metodologias.

\section{INTRODUÇÃO}

O objetivo prático e especifico deste trabalho foi aplicar as três metodologias com a finalidade de complementar e aumentar a quantidade de informações acerca das características da subsuperfície, e dar mais opções para as interpretações geológicas-sísmicas dos resultados obtidos. Os principais resultados são modelos de distribuição de velocidades, e seções empilhadas e migradas, discutidas de forma mais detalhada na sequência do trabalho.

Para o processamento, inversão e imageamento foram utilizados os aplicativos CWP/Un*x (Forel et al., 2005), crsstack (Mann, 2003), crsstack_supergathers (Baykulov, 2008) e niptomo (Duveneck, 2004).

\section{METODOLOGIA/PROBLEMA INVESTIGADO}

A metodologia do empilhamento NMO é baseada no modelo de camadas plano-horizontais, cujo tempo de trânsito para as reflexões primárias é dado por uma apro- ximação hiperbólica dada por Yilmaz (1988):

$$
t^{2}(x)=t^{2}(0)+\frac{x^{2}}{v_{\text {NMO }}^{2}},
$$

onde $x$ é o afastamento fonte-receptor, $t(x)$ o tempo de trânsito, $t(0)$ o tempo-duplo vertical simples e $v_{\mathrm{NMO}}$ a velocidade de sobretempo normal. Necessita-se de um modelo de distribuição de velocidades obtido através da marcação de pares $\left(v_{\mathrm{NMO}}, t_{0}\right)$ no mapa de coerência semblance, e que serve à correção, ao empilhamento e a migração.

O empilhamento CRS, descrito inicialmente por Müller (1999) e Mann (2002), é baseado no modelo de camadas limitadas por interfaces curvas, e considerado independente do modelo de velocidade, onde o tempo de trânsito para reflexões primárias é dado pela aproximação hiperbólica:

$$
\begin{aligned}
& t_{\mathrm{CRS}}^{2}\left(x_{m}, h\right)=\left[t(0)+2 \frac{\sin \beta_{0}}{v_{0}}\left(x_{m}-x_{0}\right)\right]^{2} \\
& +2 t(0) \frac{\cos ^{2} \beta_{0}}{v_{0}}\left[\frac{\left(x_{m}-x_{0}\right)^{2}}{R_{\mathrm{N}}}+\frac{h^{2}}{R_{\mathrm{NIP}}}\right]^{2}
\end{aligned}
$$

sendo $x_{m}$ o ponto-médio-comum, $v_{0}$ a velocidade próxima ao ponto de emergência e $h$ o meio-afastamento fonte-receptor. Neste método se recupera o trio de atributos das duas ondas hipotéticas N e NIP através da solução de um problema de otimização triparamétrica não-linear, e que são: o ângulo de emergência $\beta_{0}$, o raio de curvatura $R_{\mathrm{N}}$ e o raio de curvatura $R_{\mathrm{NIP}}$. Estes atributos carregam informações sobre a inclinação, a profundidade e a forma das interfaces refletoras. Com o objetivo de melhorar estes resultados se optou pela utilização do processo de empilhamento CRS-parcial descrito por Baykulov \& Gajewski (2008), e que produziu seções de melhor razão sinal/ruído. Este processo consta da interpolação de famílias CMP ao cubo de dados com o objetivo de densificar a malha previamente ao empilhamento CRS sob a condição de super-famílias.

A inversão tomográfica da onda NIP, desenvolvida por Duveneck (2004), visa estabelecer um mapa de distribuição de velocidades com variação lateral, para servir ao imageamento em profundidade, e utiliza os atributos CRS através dos dados marcados na seção ZO empilhada. O problema é de ajuste de curva não-linear no 
sentido dos quadrados-mínimos entre as marcações dos parâmetros dos dados de entrada $\mathbf{d},\left[\tau_{0}, \mathrm{M}_{\mathrm{NIP}}^{(\xi)}, \mathrm{p}^{(\xi)}, \xi_{0}\right]$, e as correspondentes quantidades do modelo direto $\mathbf{m}$, $[x, z, \theta]^{(\mathrm{NIP})}$, associadas ao ponto NIP do modelo. Tal desajuste é minimizado pela função custo na forma:

$S(\mathbf{m})=\frac{1}{2} \Delta \mathbf{d}^{T}(\mathbf{m}) \mathbf{C}_{\mathrm{D}}^{-1} \Delta \mathbf{d}(\mathbf{m})+\frac{1}{2} \varepsilon^{\prime \prime} \mathbf{m}^{(v) T} \mathbf{D}^{\prime \prime} \mathbf{m}^{(v)}$,

onde $\Delta \mathbf{d}(\mathbf{m})=\mathbf{d}-\mathbf{f}(\mathbf{m})$ é o vetor desvio dos dados, $\mathrm{C}_{\mathrm{D}}$ a matriz covariância dos dados, $\mathbf{D}^{\prime \prime}$ a matriz da regularização, $\varepsilon^{\prime \prime}$ o fator de ponderação entre a primeira parte que mede o ajuste de curvas e a segunda parte que mede o nível de regularização do problema, e $\mathbf{m}^{(v)}$ o vetor modelo de velocidade.

\section{RESULTADOS}

Os parâmetros e aberturas de operadores escolhidos para o processamento desta linha, assim como a ordem das etapas explicitas nos fluxogramas, foram obtidas por meio de exaustivos testes, e os resultados mais satisfatórios no sentido de maior razão sinal/ruído tem seus principais resultados expostos na sequência.

A etapa do pré-processamento, realizada por Lima (2010), foi comum a ambas metodologias, e constou dos processos mostrados na Figura 1. Para a inserção da geometria houve a análise do relatório de campo, e as principais informações encontram-se na Tabela 1.

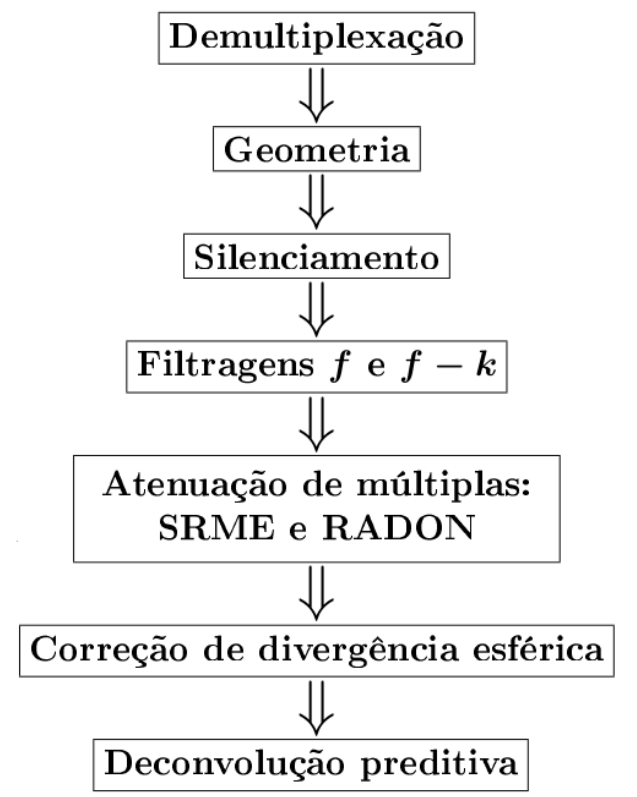

Figura 1: Fluxograma de pré-processamento.

O dado de saída desta primeira etapa, organizado em família CMP, serviu de entrada para os empilhamentos NMO e CRS. Dessa forma, o processamento NMO foi realizado e as etapas são mostradas na Figura 2.

\begin{tabular}{|c|c|}
\hline \multicolumn{2}{|c|}{ Informações da geometria de aquisição } \\
\hline \hline Bacia & Jequitinhonha \\
\hline Linha & L2140266 \\
\hline Ano de aquisição & 1985 \\
\hline Configuração & Arrasto-de-cabo \\
\hline Tipo de fonte & Canhão de ar \\
\hline Profundidade do cabo & $9 \mathrm{~m}$ a $11 \mathrm{~m}$ \\
\hline Profundidade da fonte & $9 \mathrm{~m}$ \\
\hline Comprimento do cabo & $2975 \mathrm{~m}$ \\
\hline Número de tiros & 1589 \\
\hline Número de receptores & 120 \\
\hline Intervalo entre tiros & $25 \mathrm{~m}$ \\
\hline Intervalo entre receptores & $25 \mathrm{~m}$ \\
\hline Afastamento mínimo & $150 \mathrm{~m}$ \\
\hline Afastamento máximo & $3125 \mathrm{~m}$ \\
\hline Tempo de registro & $7.0 \mathrm{~s}$ \\
\hline Intervalo de amostragem & $4 \mathrm{~ms}$ \\
\hline
\end{tabular}

Tabela 1: Informações da geometria de aquisição.

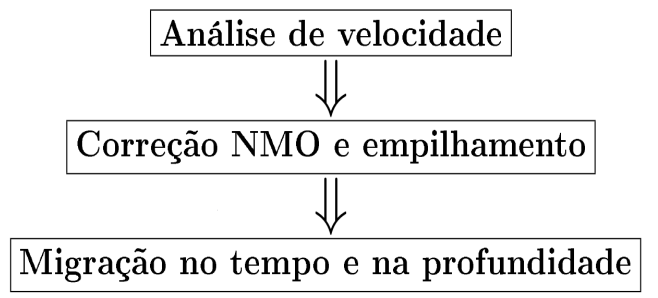

Figura 2: Fluxograma do empilhamento NMO.

Os modelos de velocidade suavizados, no tempo e na profundidade, são mostrados nas Figuras 3 e 4, e foram obtidos, respectivamente, através de marcações no mapa semblance e da conversão de velocidades médiaquadráticas para intervalares.

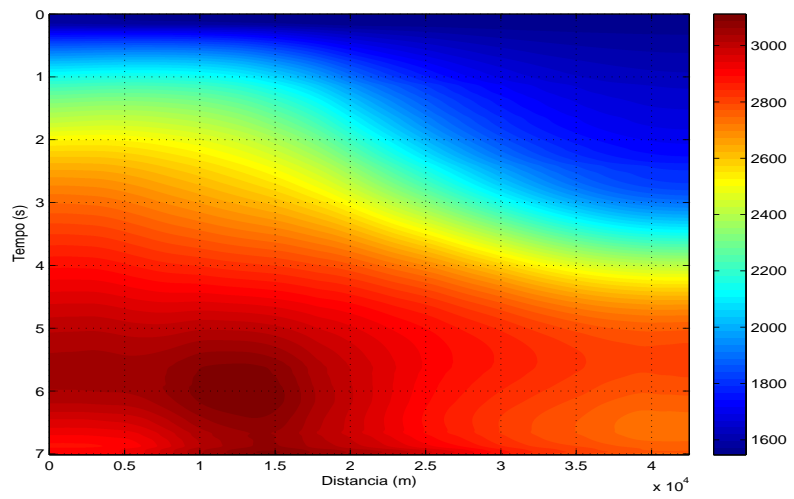

Figura 3: Modelo de velocidade semblance no tempo. 


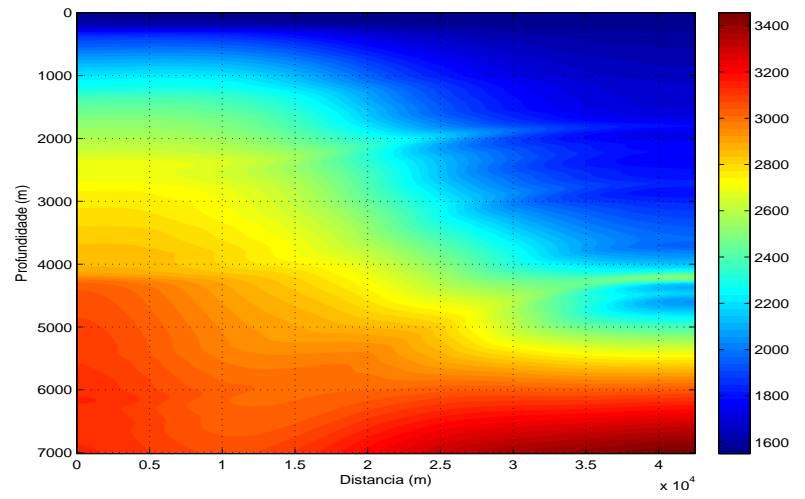

Figura 4: Modelo de velocidade semblance convertido para profundidade.

Após a correção NMO foi feito o empilhamento NMO do dado cujo resultado é mostrado na Figura 5.

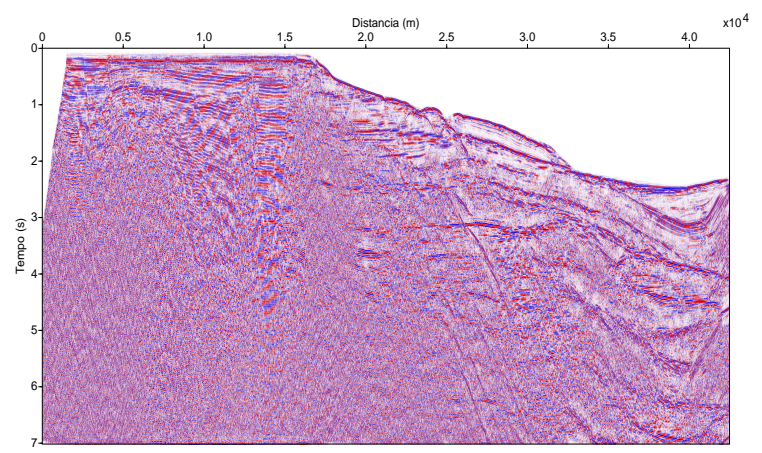

Figura 5: Seção empilhada NMO.

Nesta figura se observa que a maior parte da informação registrada corresponde a eventos rasos e com boa continuidade. No entanto se observam muitas difrações a medida que a profundidade aumenta.

Os resultados da migração Kirchhoff pós-empilhamento, no tempo e na profundidade, são mostrados nas Figuras 6 e 7 , respectivamente.

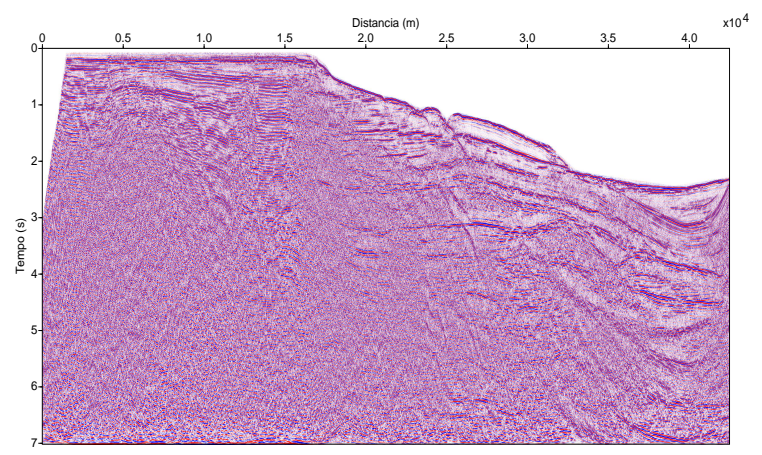

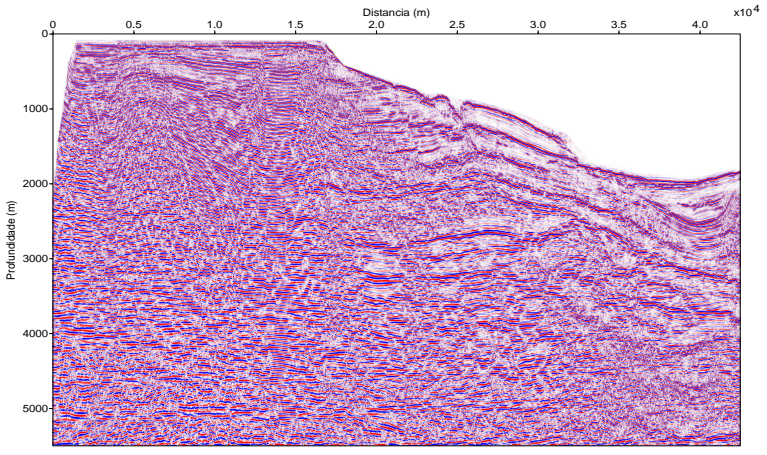

Figura 7: Seção migrada Kirchhoff na profundidade.

Analisando as Figuras 6 e 7 se observa o pequeno deslocamento das estruturas geológicas em relação à Figura 5 , a recuperação da subhorizontalização das estruturas, desenhada pela melhor continuidade dos refletores, o colapso das difrações e a correção das falhas geológicas. A seção 7 possui qualidade superior do que a Figura 6 devido a recuperação de eventos em grandes profundidades e a ausência de arcos sobre os pontos difratores ("sorrisos").

O processo de densificação do dado, denominado Empilhamento CRS Parcial (Ver Figura 8), realizado inicialmente por Lima (2010) e complementado por Silva (2012), tem por saída o dado organizado em superfamílias CMP, e que é entrada para o empilhamento CRS, cujas etapas são mostradas na Figura 9.

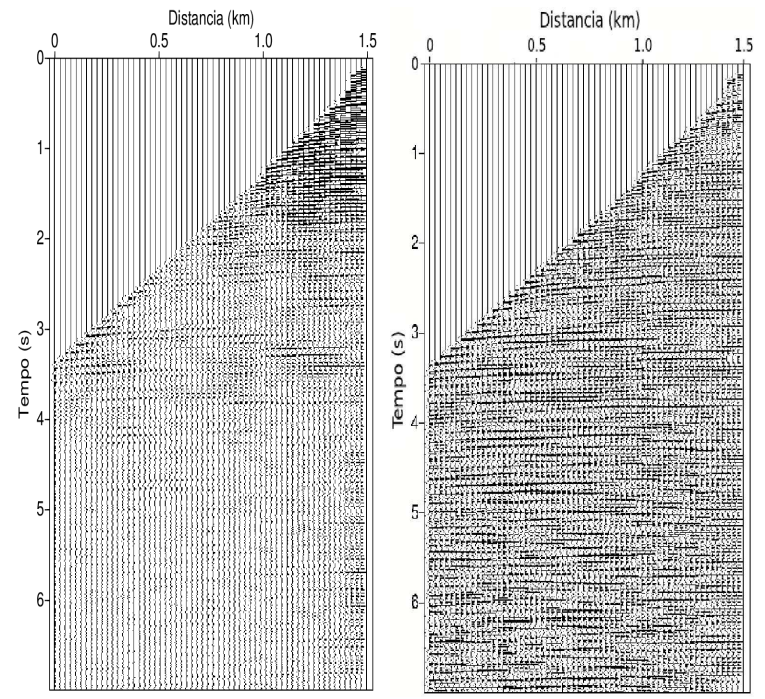

Figura 8: Família CMP 1010 antes e depois da densificação do dado pré-empilhado.

Figura 6: Seção migrada Kirchhoff no tempo. 


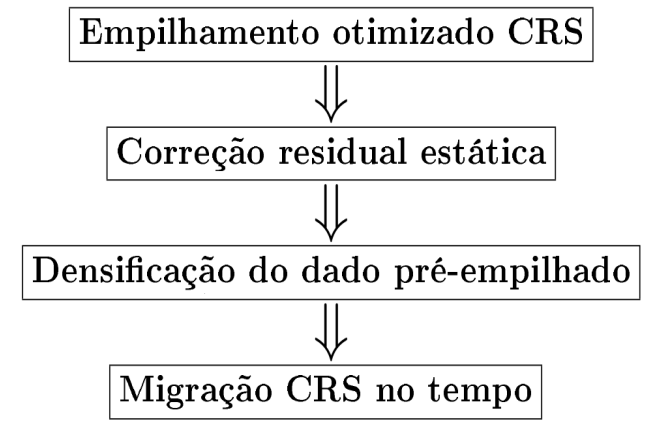

Figura 9: Fluxograma do empilhamento CRS.

Seguindo com o fluxograma de empilhamento CRS, foram selecionados sub-produtos, como as seções CRS empilhada (ver Figura 10) e migrada (ver Figura 11) submetidas à Primeira Zona de Fresnel Projetada.

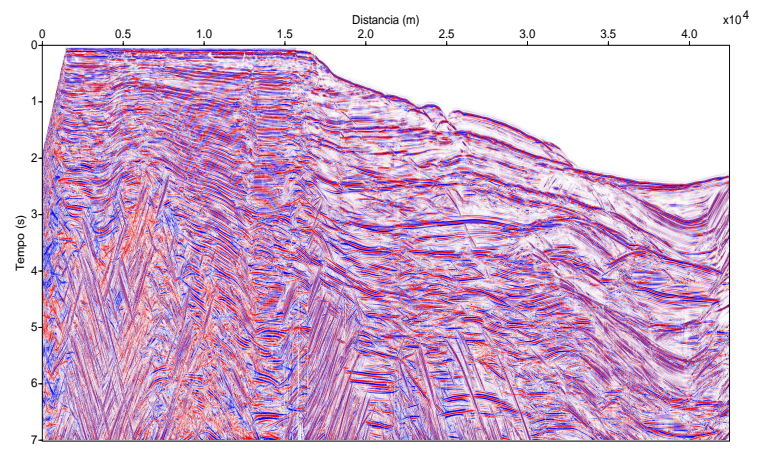

Figura 10: Seção empilhada CRS.

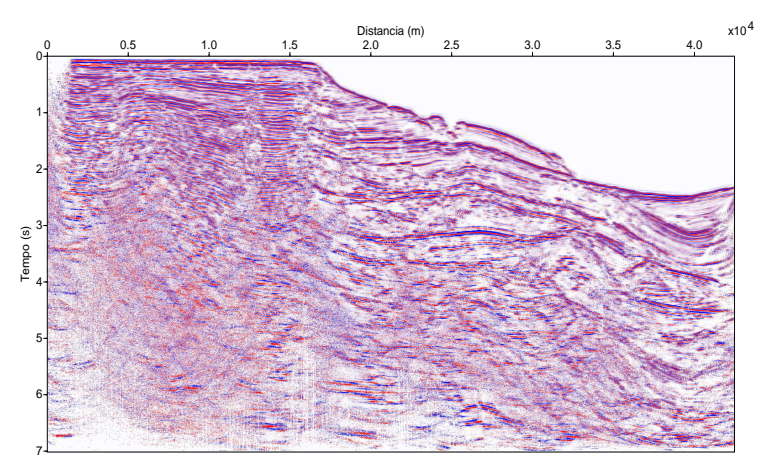

Figura 11: Seção CRS-migrada.

A Figura 12 mostra a migração Kirchhoff no tempo utilizando o modelo de velocidade da Figura 3 e a seção empilhada CRS otimizada da Figura 10.

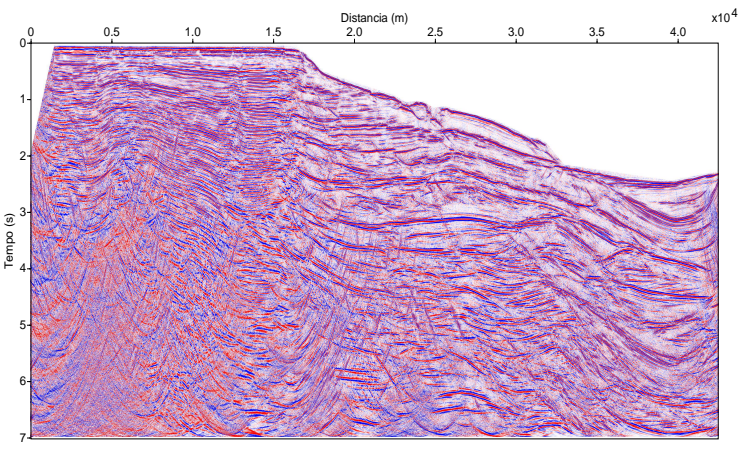

Figura 12: Migração Kirchhoff usando o modelo de velocidade semblance e o empilhamento CRS.

A seção empilhada CRS apresenta melhor qualidade visual do que a NMO no sentido de realçar mais as estruturas devido à melhor continuidade dos eventos refletores e a grande quantidade de difrações. O realce da múltipla de superfície livre também é maior, como a coerência mais acentuada para os eventos de reflexão primária.

Sobre a migração CRS, se observa o colapso das difrações, a correção das falhas geológicas, a continuidade dos eventos refletores, a grande quantidade de granulações, a ausência de arcos sobre os pontos difratores ("sorrisos") e a atenuação da múltipla de superfície livre.

A migração Kirchhoff no tempo, utilizando o modelo de velocidade semblance, e a seção empilhada CRS mostra muitas difrações e uma grande quantidade de arcos acima dos pontos difratores ("sorrisos"), principalmente para eventos em grandes profundidades, demonstrando a baixa qualidade da seção e a incoerência da metodologia.

$\mathrm{Na}$ inversão tomográfica da onda NIP, cujo fluxograma está mostrado na Figura 13, foram feitas 2970 marcações no domínio $\left(\tau_{0}, \xi_{0}\right)$ sobre os eventos de reflexões primárias da seção empilhada CRS (ver Figura 14). 


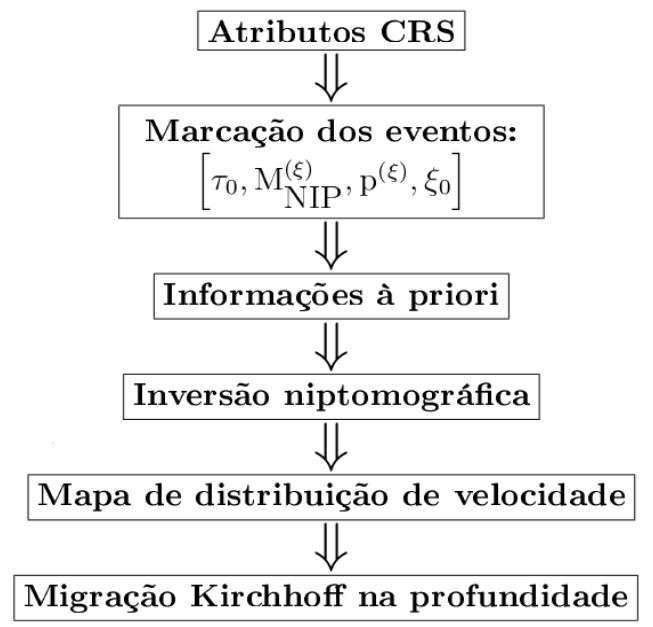

Figura 13: Fluxograma da inversão niptomográfica.

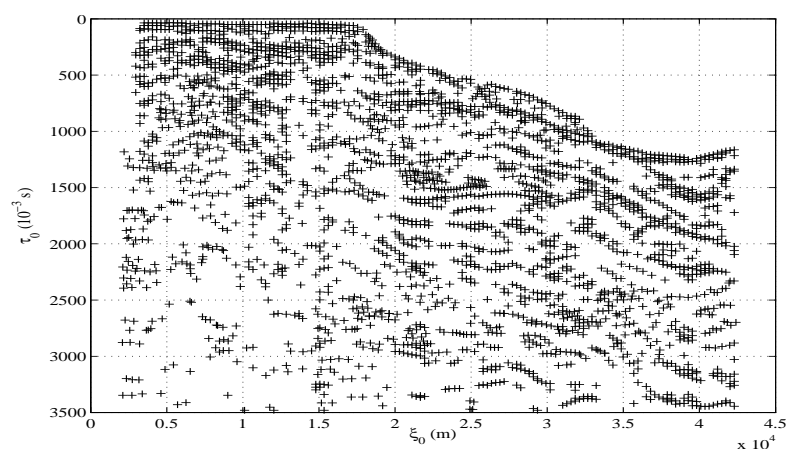

Figura 14: Mapa das 2970 marcações feitas sobre a seção empilhada CRS.

A Figura 15 mostra a minimização da função custo em função do número de iterações, onde se observa a redução do valor do desajuste entre os parâmetros dos dados de entrada e do modelo.

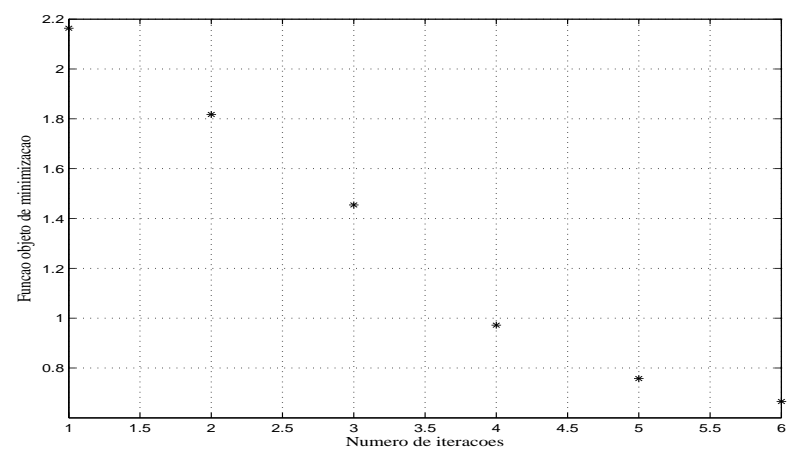

Figura 15: Minimização da função desajuste em função do número de iterações.

Os modelos de velocidade niptomográfico inicial e final, são mostrados, respectivamente, nas Figuras 16 e 17, onde se observa a grande evolução do modelo final.

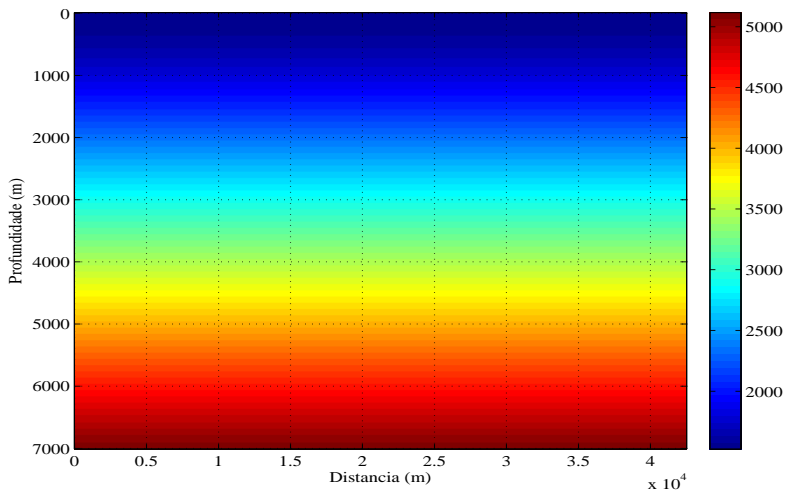

Figura 16: Modelo de velocidade niptomográfico inicial.

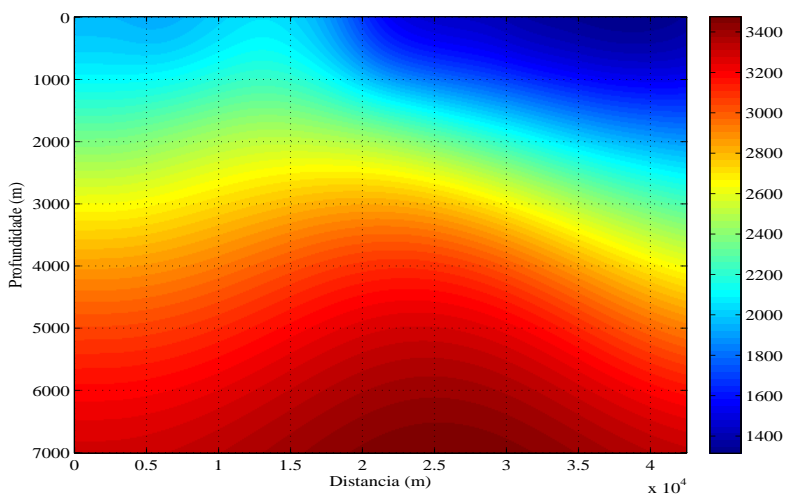

Figura 17: Modelo de velocidade niptomográfico final.

Utilizando o modelo da Figura 17 foi realizada a migração Kirchhoff em profundidade, e o resultado é mostrado na Figura 18.

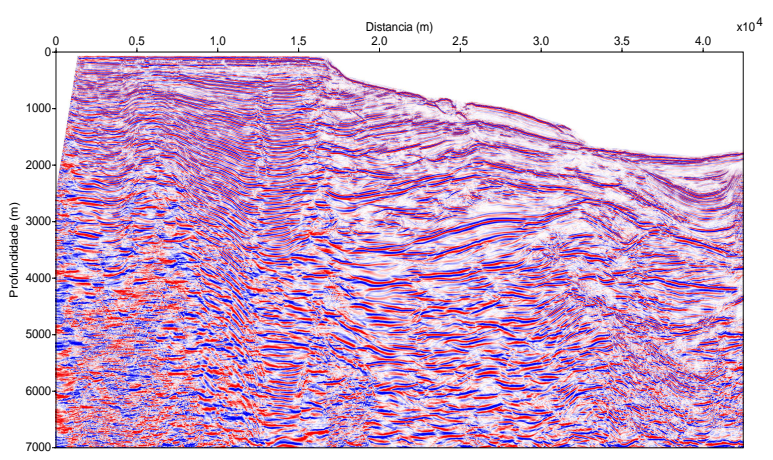

Figura 18: Seção migrada Kirchhoff na profundidade.

O modelo de velocidade niptomográfico, além de possuir grande semelhança com o modelo de velocidade semblance (ver Figura 4), mostra com melhor definição o fundo oceânico, a distribuição dos valores de maiores velocidades nas regiões mais profundas da seção geológica e as curvas de nível com os diferentes contrastes de 
velocidade.

Na migração Kirchhoff se observa o colapso das difrações nas regiões mais profundas da bacia e a ausência de arcos acima dos pontos difratores ("sorrisos").

\section{DISCUSSÃO E CONCLUSÕES}

As conclusões sobre os resultados dos empilhamentos NMO e CRS, da inversão niptomográfica e da migração Kirchhoff, são claramente mostrando dificuldades e limitações sérias, bem como casos que merecem mais atenção quanto a implementação das teorias estudadas, e servem de referência para trabalhos futuros.

Do ponto de vista comparativo entre as seções obtidas, a seção empilhada CRS possui resolução vertical e horizontal superior à seção empilhada $\mathrm{NMO}$, uma vez que na seção CRS se pode destinguir melhor as interfaces refletoras e por evidenciar estruturas contínuas atraves da seção, além de destacar de forma mais clara os padrões de difração. A comparação entre as seções migradas dessas duas metodologias mostra que a migração-CRS possui melhor qualidade visual e continuidade dos eventos refletores.

Com relação aos modelos de velocidade se pôde concluir que o obtido através da niptomografia possui melhor qualidade visual do que o modelo de velocidade semblance, devido sua melhor distribuição dos valores na seção geológica, evidenciando a coerência da metodologia.

A comparação entre os resultados da migração Kirchhoff na profundidade mostra que a seção obtida utilizando o modelo de velocidade niptomográfico possui qualidade superior à respectiva seção obtida através do modelo de velocidade semblance, uma vez que há grande diferença na continuidade dos eventos refletores e no colapso das difrações.

Como sugestões para trabalhos futuros, se propõe a aplicação de outras técnicas de migração pós-empilhamento na profundidade baseadas na equação da onda acústica, como as metodologias PSPI (Phase-Shift Plus Interpolation), SS (Split-Step), RTM (Reverse Time Migration), e FFD (Fourier Finite Difference), para efeito de comparação com a migração Kirchhoff, e a generalização dos resultados obtidos com as técnicas CRS e niptomográfica para o caso 3D.

\section{AGRADECIMENTOS}

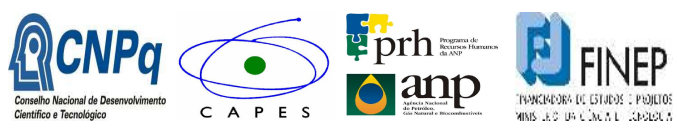

Baykulov, M., and Gajewski, D., 2008, Prestack seismic data enhancement with partial common reflection surface (crs) stack: Wave Inversion Technology, , no. $1,57-73$.

Baykulov, M., 2008, Partial crs stack user's manual: University of Hamburg.

Duveneck, E., 2004, Tomographic determination of seismic velocity models with kinematic wavefield attributes: Universität Karlsruhe.

Forel, D., Benz, T., and Pennington, W. D., 2005, Seismic data processing with seismic un $^{*} \mathrm{x}$ - a $2 \mathrm{~d}$ seismic data processing primer:, volume 12 Society of Exploration Geophysicists.

Lima, H. M., 2010, Processamento e imageamento de dados sísmicos marinhos: Universidade Federal do Pará.

Mann, J., 2002, Extensions and applications of the common-reflection-surface stack method: Universität Karlsruhe.

Mann, J., 2003, 2-d commom-reflection-surface stack user's manual: Universität Karlsruhe.

Müller, T., 1999, The commom reflection surface method - seismic imaging without explicit knowledge of the velocity model: Universität Karlsruhe.

Silva, D. A. B., 2012, Processamento, inversão e imageamento de dados sísmicos marinhos: Universidade Federal do Pará.

Yilmaz, O., 1988, Seismic data processing:, volume 2 Society of Exploration Geophysicists.

\section{REFERÊNCIAS}

